\title{
A Noncovalent Switch for Lysozyme
}

Kirstin Wenck, Sebastian Koch, Christian Renner, Wei Sun, Thomas Schrader

Corresponding author: Thomas Schrader, Institute of Organic Chemistry, Universität Duisburg-Essen, Universitätsstr. 5, 45117 Essen; e-mail:Thomas.Schrader@uni-due.de

\section{Supporting Information}

\section{Table of Contents}

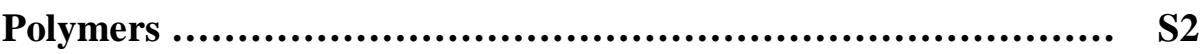

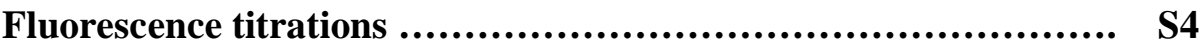

Fluorescence spectra .......................................... S6

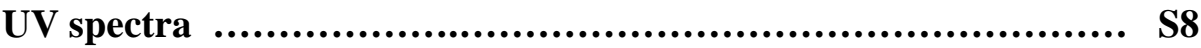

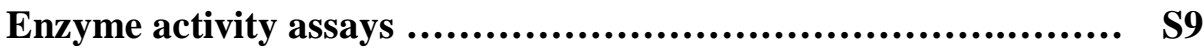

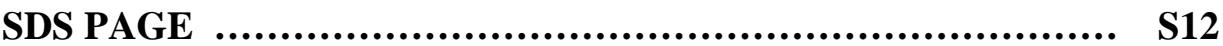

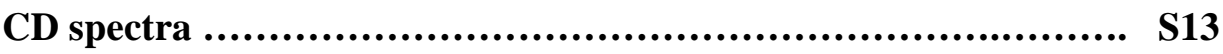

Gel electrophoresis............................................... S15 


\section{Polymers.}

Polymer 1, 4 - 6: Sebastian J. Koch, Christian Renner, Xiulan Xie, Thomas Schrader, Angew.

Chem. Int. Ed. 2006, 45, 6352.

\section{Polymer 2,3:}

\begin{tabular}{|l|l|l|}
\hline & Polymer 2 & Polymer 3 \\
\hline bisphosphonate & $675 \mathrm{mg}(1.665 \mathrm{mmol}, 3 \mathrm{eq})$ & \\
\hline sugaramide & & $428 \mathrm{mg}(1.73 \mathrm{mmol}, 1 \mathrm{eq})$ \\
\hline alkylamide & $141 \mathrm{mg}(0.555 \mathrm{mmol}, 1 \mathrm{eq})$ & \\
\hline hydroxylamide & $79 \mathrm{mg}(0.555 \mathrm{mmol}, 1 \mathrm{eq}$ & \\
\hline dansylamide & $103 \mathrm{mg}(0.228 \mathrm{~mol}, 8 \mathrm{~mol} \%)$ & $50 \mathrm{mg}(0.11 \mathrm{mmol}, 4 \mathrm{~mol} \%)$ \\
\hline methylmethacrylate & & $175 \mathrm{mg}(1.75 \mathrm{mmol}, 1 \mathrm{eq})$ \\
\hline AIBN & $3 \mathrm{mg}(0.0183 \mathrm{mmol}, 1 \mathrm{~mol} \%)$ & $8,67 \mathrm{mg}(0.05 \mathrm{mmol}, 2 \mathrm{~mol} \%)$ \\
\hline DMF & $7 \mathrm{~mL}$ & $4 \mathrm{~mL}$ \\
\hline duration & $22 \mathrm{~h}$ & $24 \mathrm{~h}$ \\
\hline Yield & $187 \mathrm{mg}(8 \%)$ & $239 \mathrm{mg} \mathrm{(37} \mathrm{\% )}$ \\
\hline
\end{tabular}

Integrations represent number of equivalent protons multiplied with the stoichiometric factor in the copolymer.

${ }^{1}$ H-NMR Polymer 2a (300 MHz, CD $\left.{ }_{3} \mathrm{OD}\right): \delta[\mathrm{ppm}]=0.83-0.95(\mathrm{~m}, 3 \mathrm{H}), 0.99-1.51(\mathrm{~m}, 28$ H), 3.05-3.39 (m, 6 H), 3.50-3.79 (m, 44 H), 6.92-7.08 (m, 3 H), 7.18-7.59 (m, 6 H), 8.10$8.60(\mathrm{~m}, 1 \mathrm{H})$.

${ }^{1}$ H-NMR Polymer $3\left(300 \mathrm{MHz}, \mathrm{D}_{2} \mathrm{O}\right): \delta[\mathrm{ppm}]=0.76-2.53(\mathrm{~m}, 6 \mathrm{H}), 3.28-4.04(\mathrm{~m}, 14 \mathrm{H})$, 5.08-5.28 (m, $1 \mathrm{H})$. 


\section{Polymer-analogous cleavage of phosphonic acid methyl esters in polymer 2:}

$180 \mathrm{mg}$ polymer 2 were dissolved in $10 \mathrm{~mL}$ absolute acetonitrile under an argon atmosphere. $1.4 \mathrm{~g}$ lithium bromide solution $(61 \mathrm{mg}, 0.702 \mathrm{mmol}, 2.2 \mathrm{eq}$ for each bisphosphonic acid tetramethyl ester present in the polymer, $4.52 \%$ in acetonitrile) were added. The clear solution is stirred under argon for 72 hours at $90{ }^{\circ} \mathrm{C}$. During this period the polyanionic product precipitates as poly-(lithiumphosphonate) salt from the reaction mixture. The solvent is decanted and the yellowish solid is washed three times with acetonitrile. $126 \mathrm{mg}$ of pure product $(69 \%)$ is obtained after filtration and drying in vacuo.

${ }^{1}$ H-NMR Polymer 2b (300 MHz, CD $\left.3 \mathrm{OD}\right): \delta[\mathrm{ppm}]=0.54-1.54(\mathrm{~m}, 18 \mathrm{H}), 3.52-3.15(\mathrm{~m}, 5$ H), 3.23-3.68 (m, $23 \mathrm{H}), 6.84-7.18(\mathrm{~m}, 9 \mathrm{H})$.

${ }^{31}$ P-NMR Polymer 2b (300 MHz, $\left.\mathrm{CD}_{3} \mathrm{OD}\right): \delta[\mathrm{ppm}]=22.09(\mathrm{~s})$.

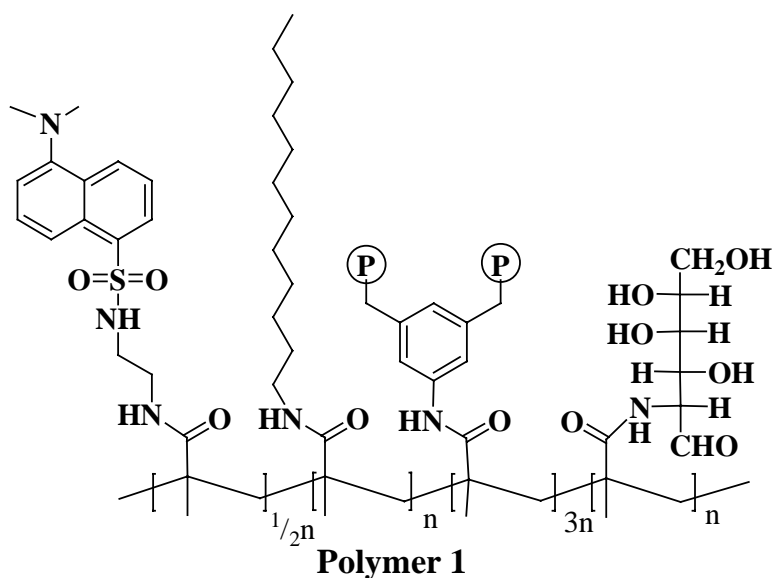

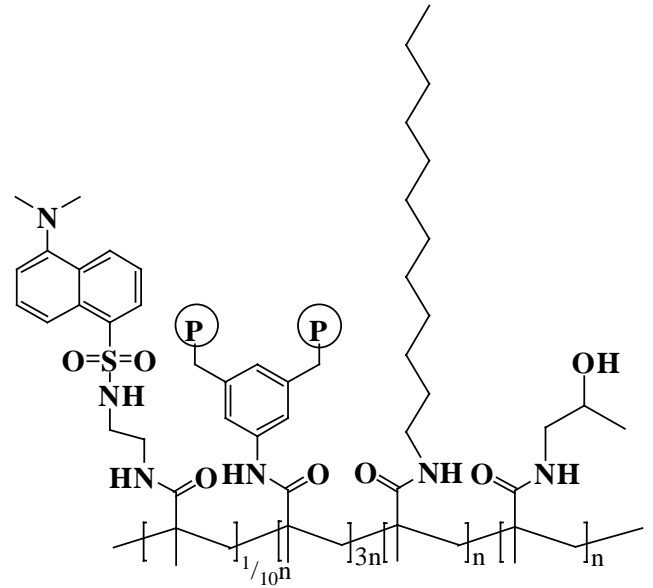

Polymer 2

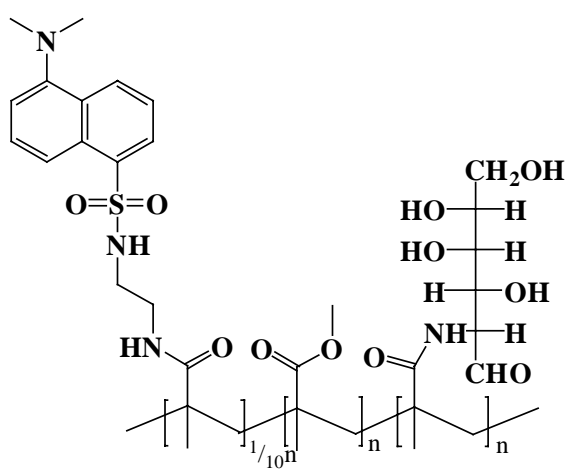

Polymer 3 


\section{Fluorescence titrations.}

Polymer $2 \boldsymbol{b}$ vs. Lysozyme

c $($ polymer $\mathbf{2 b})=7.6 \cdot 10^{-6} \mathrm{~mol} / \mathrm{L}$

stoichiometry polymer : Lysozyme $=1: 1$

$\mathrm{K}_{\mathrm{a}}=1.9 \cdot 10^{7} \mathrm{M}^{-1} \pm 51 \%$

\begin{tabular}{|c|c|c|c|}
\hline c (Lysozyme) [mol/L] & equivalents & $\mathrm{I}_{520 \mathrm{~nm}}$ & $\Delta \mathrm{I}_{520 \mathrm{~nm}}$ \\
\hline $0.000 \mathrm{E}+00$ & 0.000 & 141.343 & 0 \\
\hline $2.834 \mathrm{E}-07$ & 0.038 & 144.878 & 3.535 \\
\hline $6.786 \mathrm{E}-07$ & 0.090 & 147.933 & 6.59 \\
\hline $1.025 \mathrm{E}-06$ & 0.136 & 153.656 & 12.313 \\
\hline $1.584 \mathrm{E}-06$ & 0.210 & 160.237 & 18.894 \\
\hline $2.260 \mathrm{E}-06$ & 0.299 & 168.583 & 27.24 \\
\hline $3.230 \mathrm{E}-06$ & 0.428 & 179.427 & 38.084 \\
\hline $4.191 \mathrm{E}-06$ & 0.555 & 194.155 & 52.812 \\
\hline $5.437 \mathrm{E}-06$ & 0.720 & 209.464 & 68.121 \\
\hline $6.874 \mathrm{E}-06$ & 0.910 & 222.655 & 81.312 \\
\hline $8.435 \mathrm{E}-06$ & 1.117 & 233.01 & 91.667 \\
\hline $1.003 \mathrm{E}-05$ & 1.328 & 237.414 & 96.071 \\
\hline $1.166 \mathrm{E}-05$ & 1.544 & 236.651 & 95.308 \\
\hline $1.328 \mathrm{E}-05$ & 1.759 & 236.475 & 95.132 \\
\hline $1.485 \mathrm{E}-05$ & 1.967 & 234.719 & 93.376 \\
\hline
\end{tabular}

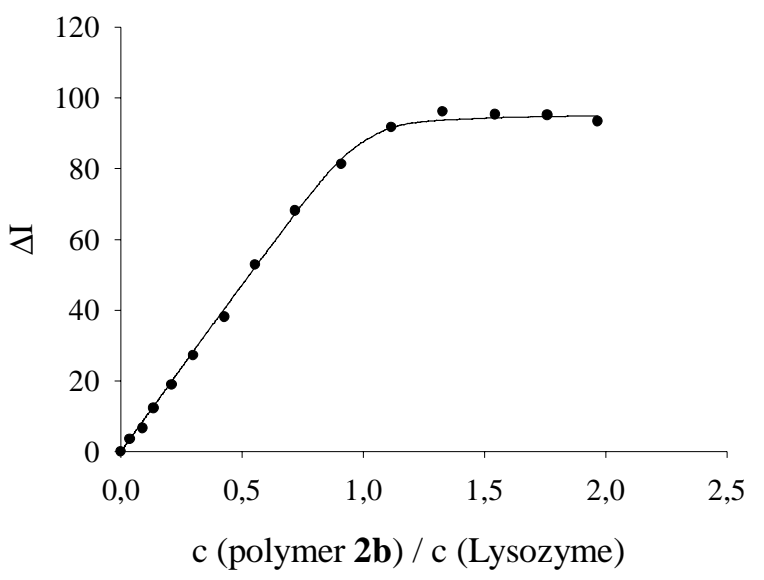

Beyond 2 eqs. of added polymer the complex began to precipitate and prevented further extension of the stoichiometric ratio as well as produced relatively large errors (see above). 
$\mathrm{c}\left(\right.$ polymer 3) $=6.6 \cdot 10^{-6} \mathrm{~mol} / \mathrm{L}$

no binding

\begin{tabular}{|c|c|c|c|}
\hline c (Lysozyme) [mol/L] & equivalents & $\mathrm{I}_{520 \mathrm{~nm}}$ & $\Delta \mathrm{I}_{520 \mathrm{~nm}}$ \\
\hline $0.000 \mathrm{E}+00$ & 0.000 & 124.037 & 0 \\
\hline $2.192 \mathrm{E}-07$ & 0.033 & 123.766 & 0.271 \\
\hline $6.483 \mathrm{E}-07$ & 0.098 & 124.13 & -0.093 \\
\hline $1.106 \mathrm{E}-06$ & 0.168 & 123.86 & 0.177 \\
\hline $1.670 \mathrm{E}-06$ & 0.254 & 123.32 & 0.717 \\
\hline $2.475 \mathrm{E}-06$ & 0.376 & 122.178 & 1.859 \\
\hline $3.412 \mathrm{E}-06$ & 0.518 & 122.778 & 1.259 \\
\hline $4.515 \mathrm{E}-06$ & 0.686 & 121.35 & 2.687 \\
\hline $5.883 \mathrm{E}-06$ & 0.893 & 121.257 & 2.78 \\
\hline $7.396 \mathrm{E}-06$ & 1.123 & 122.045 & 1.992 \\
\hline $9.089 \mathrm{E}-06$ & 1.380 & 123.278 & 0.759 \\
\hline $1.083 \mathrm{E}-05$ & 1.645 & 119.844 & 4.193 \\
\hline $1.258 \mathrm{E}-05$ & 1.910 & 120.529 & 3.508 \\
\hline $1.393 \mathrm{E}-05$ & 2.116 & 120.649 & 3.388 \\
\hline $1.566 \mathrm{E}-05$ & 2.379 & 119.647 & 4.39 \\
\hline $1.727 \mathrm{E}-05$ & 2.623 & 119.626 & 4.411 \\
\hline
\end{tabular}

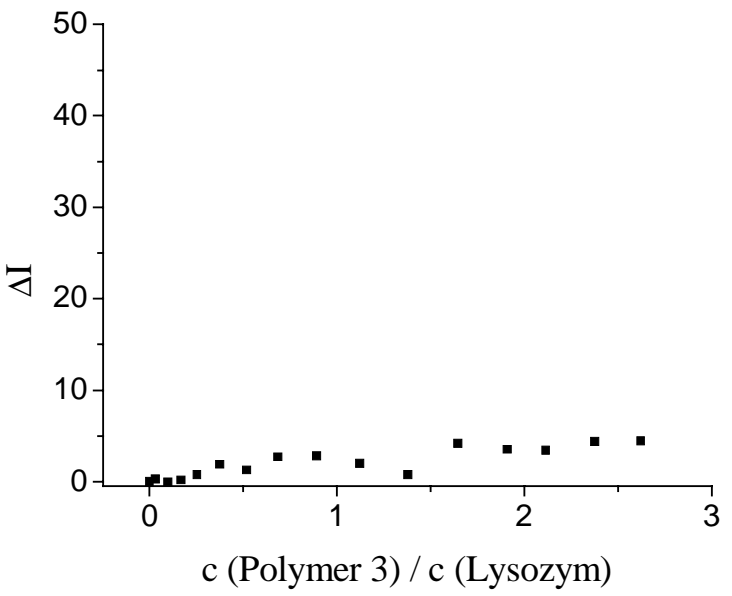




\section{Fluorescence spectra.}

Fluorescence spectra of pure lysozyme, pure copolymer and their mixtures were recorded at following concentrations:

\section{a) pure enzyme sample:}

c $($ Lysozyme $)=2.8 \cdot 10^{-5} \mathrm{~mol} / \mathrm{L}$

b) pure polymer sample:

$\mathrm{c}\left(\right.$ polymer 4) $=7.3 \cdot 10^{-6} \mathrm{~mol} / \mathrm{L}$ in $10 \mathrm{mM}$ phosphate buffer $(\mathrm{pH} 7.0)$

c) enzyme/polymer mixtures:

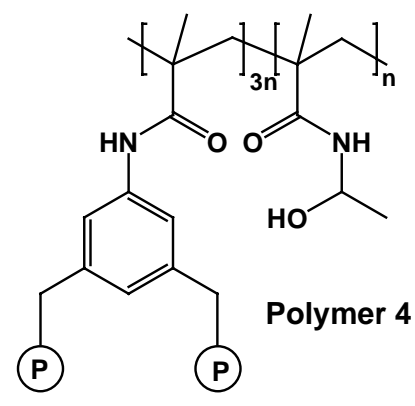

$\mathrm{c}($ polymer 4$)=7.13 \cdot 10^{-6} \mathrm{~mol} / \mathrm{L}$ in Lysozyme-solution $\left(\mathrm{c}=2.8 \cdot 10^{-5} \mathrm{~mol} / \mathrm{L}\right)$

Detection range $300-400 \mathrm{~nm}$ :

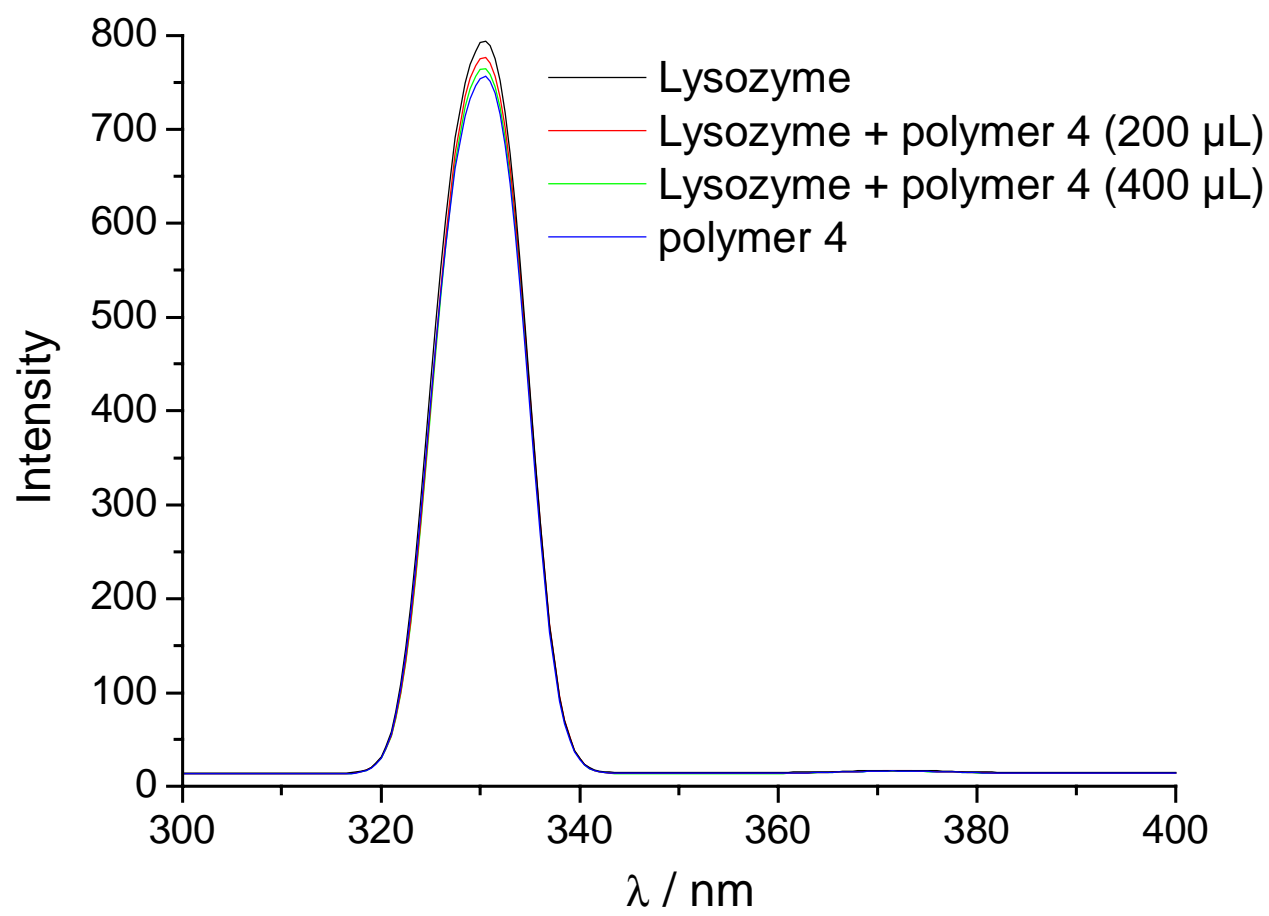

Scattered fluorescence signal at exactly $330 \mathrm{~nm}$, no additional tryptophane emission intensity at the same wavelength. 
Detection range 400-600 $\mathrm{nm}$ :

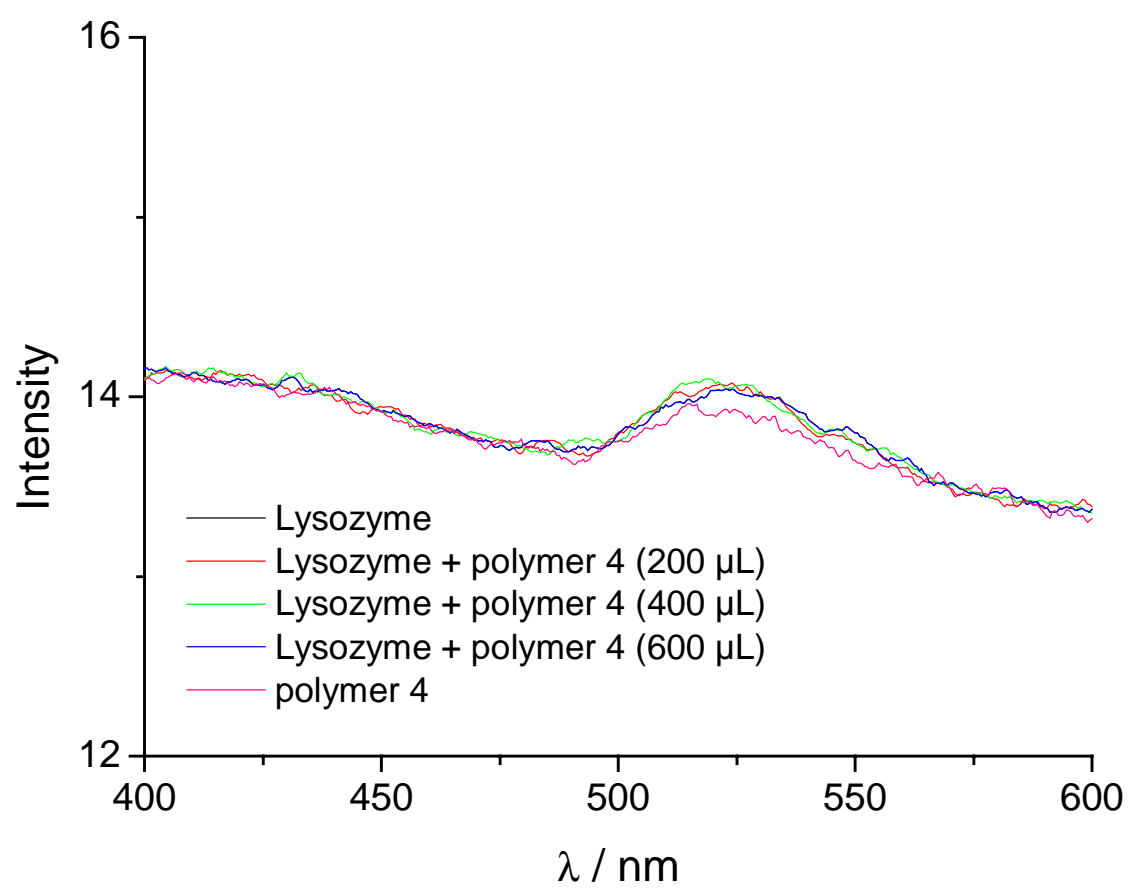

In the presence of varying concentrations of polymer $\mathbf{4}$, no additional emission intensity occurs at the detection wavelength for the Dansyl group at $540 \mathrm{~nm}$. 


\section{UV spectra.}

$\mathrm{c}($ Lysozyme $)=2.8 \cdot 10^{-5} \mathrm{~mol} / \mathrm{L}$

$\mathrm{c}($ polymer 4$)=7.13 \cdot 10^{-6} \mathrm{~mol} / \mathrm{L}$ in Lysozyme-solution $\left(\mathrm{c}=2.8 \cdot 10^{-5} \mathrm{~mol} / \mathrm{L}\right)$

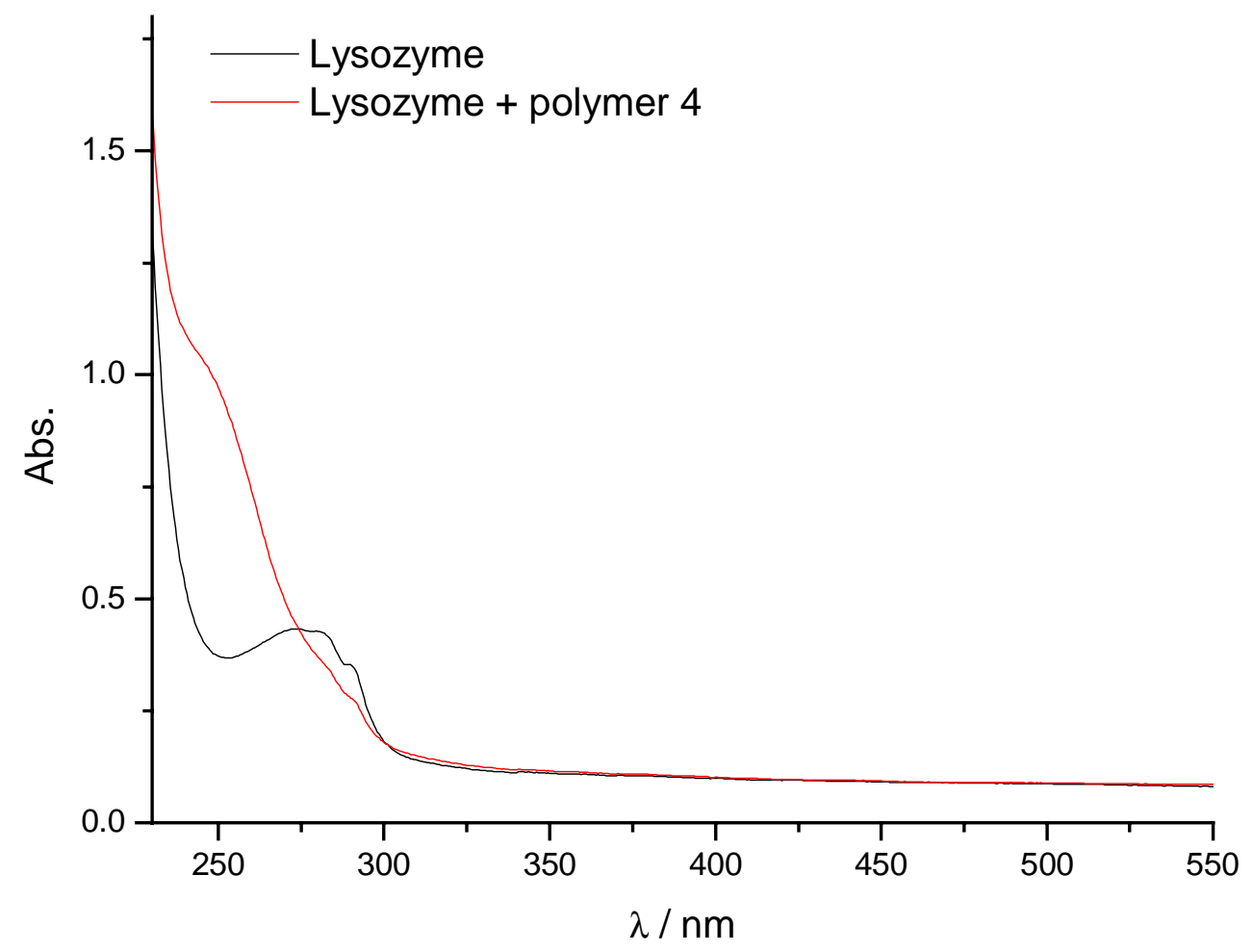

No absorption of the protein-polymer complex occurs above $300 \mathrm{~nm}$ (tryptophane absorption at $280 \mathrm{~nm}$; irradiation of Dansyl groups at $330 \mathrm{~nm}$ ). 


\section{Enzyme activity assays.}

Lysozyme vs. Polymer 1

$\mathrm{IC}_{50}=0.7 \mu \mathrm{M}$

stiochiometry: 1:1 Polymer 1:Lysozyme

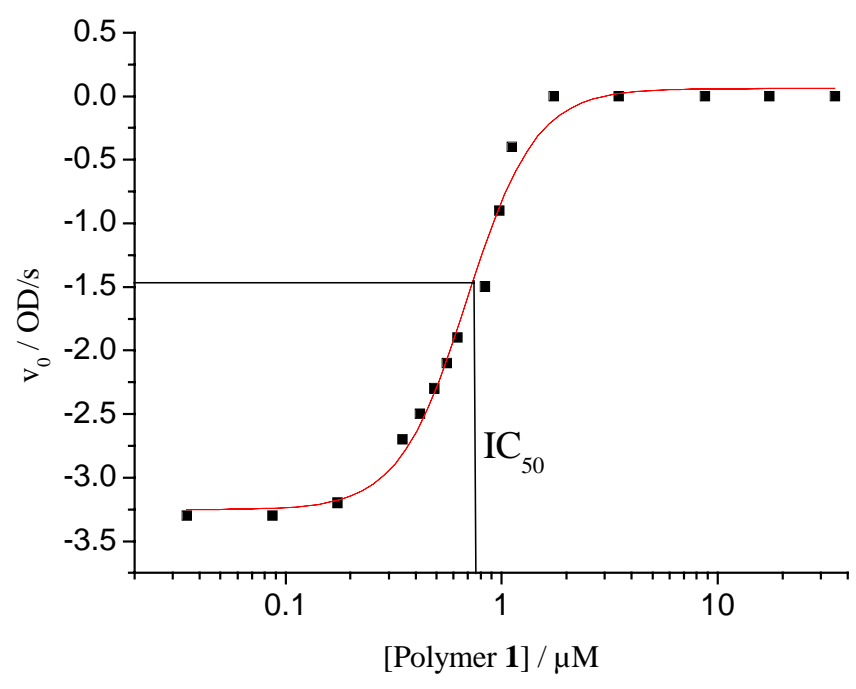

\begin{tabular}{|c|c|}
\hline$[$ Polymer 1] $/ \mu \mathrm{M}$ & $\mathrm{v}_{0} / \mathrm{OD} / \mathrm{s}$ \\
\hline 34.988 & 0 \\
\hline 17.494 & 0 \\
\hline 8.747 & 0 \\
\hline 3.499 & 0 \\
\hline 1.749 & 0 \\
\hline 1.118 & -0.4 \\
\hline 0.979 & -0.9 \\
\hline 0.839 & -1.5 \\
\hline 0.629 & -1.9 \\
\hline 0.559 & -2.1 \\
\hline 0.489 & -2.3 \\
\hline 0.419 & -2.5 \\
\hline 0.350 & -2.7 \\
\hline 0.175 & -3.2 \\
\hline 0.087 & -3.3 \\
\hline 0.035 & -3.3 \\
\hline
\end{tabular}

Lysozyme vs. Polymer $\mathbf{2 b}$

$\mathrm{IC}_{50}=6.7 \mu \mathrm{M}$

stoichiometry: 8.5:1 Polymer 2b:Lysozyme

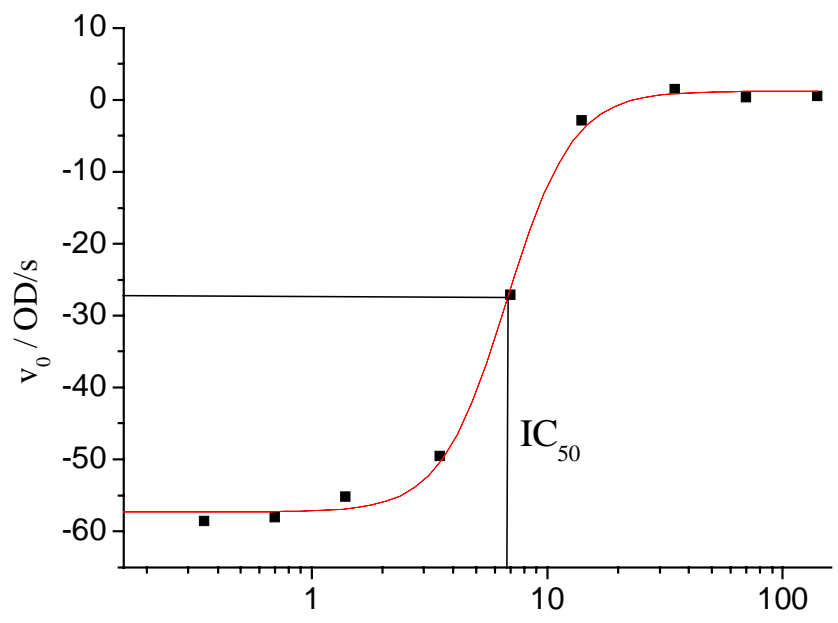

\begin{tabular}{|c|c|}
\hline$[$ Polymer $\mathbf{2 b}] / \mu \mathrm{M}$ & $\mathrm{v}_{0} / \mathrm{OD} / \mathrm{s}$ \\
\hline 139.950 & 0.50 \\
\hline 69.975 & 0.35 \\
\hline 34.988 & 1.49 \\
\hline 13.995 & -2.88 \\
\hline 6.998 & -27.17 \\
\hline 3.499 & -49.54 \\
\hline 1.400 & -55.18 \\
\hline 0.700 & -58.09 \\
\hline 0.350 & -58.56 \\
\hline 0.140 & -57.90 \\
\hline
\end{tabular}

[Polymer 2b] / $\mu \mathrm{M}$ 
Lysozyme vs. Polymer 3

no inhibition

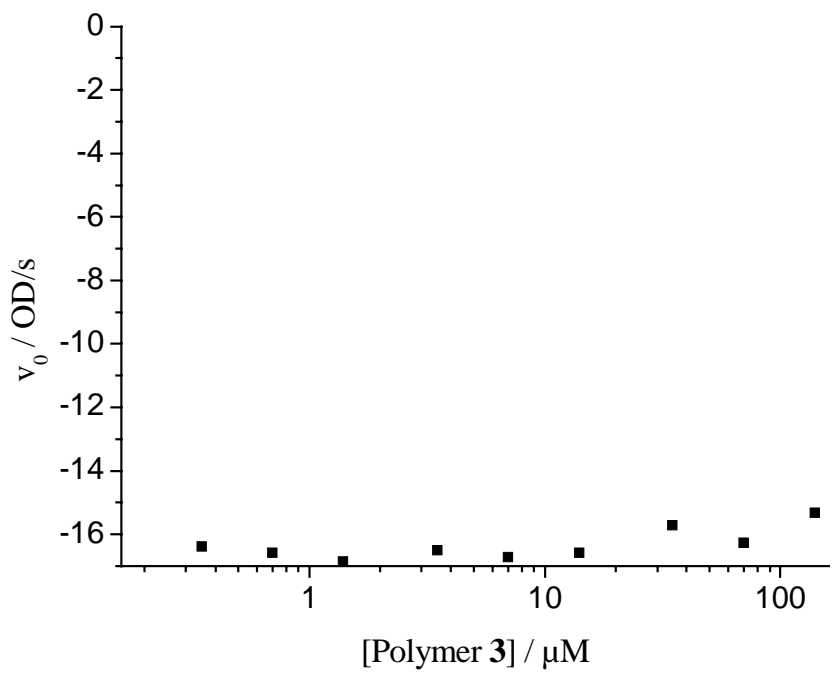

\begin{tabular}{|c|c|}
\hline$[$ Polymer 3] $/ \mu \mathrm{M}$ & $\mathrm{v}_{0} / \mathrm{OD} / \mathrm{s}$ \\
\hline 139.950 & -15.33 \\
\hline 69.975 & -16.27 \\
\hline 34.988 & -15.72 \\
\hline 13.995 & -16.59 \\
\hline 3.499 & -16.51 \\
\hline 1.400 & -16.87 \\
\hline 0.700 & -16.60 \\
\hline 0.350 & -16.40 \\
\hline 0.140 & -16.77 \\
\hline 0.070 & -16.12 \\
\hline
\end{tabular}

Lysozyme vs. Polymer 1

c $(\mathrm{NaCl})=100 \mathrm{mM}$

$\mathrm{IC}_{50}=2.3 \mu \mathrm{M}$

Stöchiometrie: 3.3:1 Polymer 1:Lysozyme

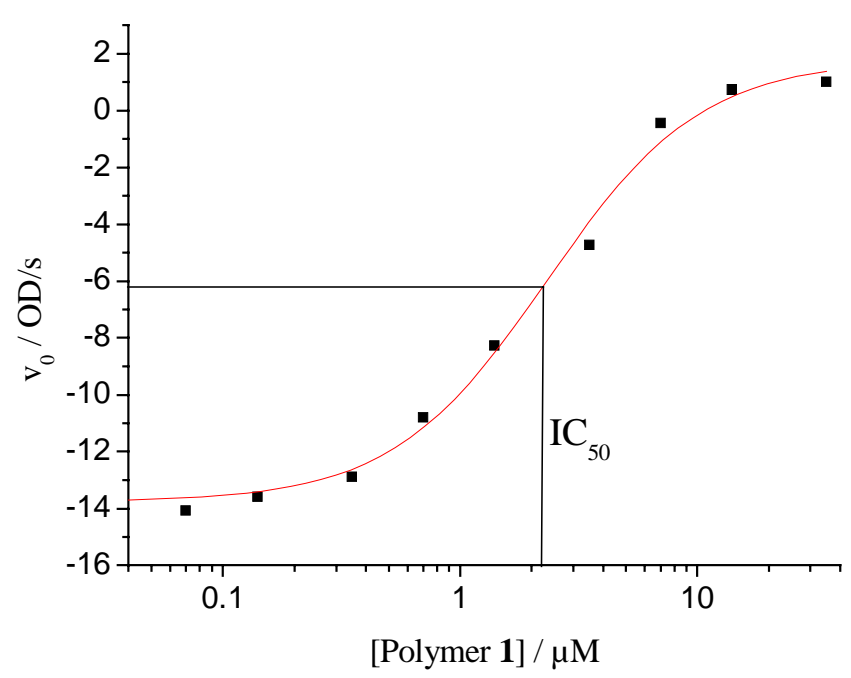

\begin{tabular}{|c|c|}
\hline [Polymer 1] $/ \mu \mathrm{M}$ & $\mathrm{v}_{0} / \mathrm{OD} / \mathrm{s}$ \\
\hline 34.988 & 1.00 \\
\hline 13.995 & 0.73 \\
\hline 6.998 & -0.45 \\
\hline 3.499 & -4.73 \\
\hline 1.400 & -8.28 \\
\hline 0.700 & -10.80 \\
\hline 0.350 & -12.89 \\
\hline 0.140 & -13.60 \\
\hline 0.070 & -14.07 \\
\hline 0.035 & -12.80 \\
\hline 0.014 & -13.83 \\
\hline 0.007 & -13.97 \\
\hline
\end{tabular}


$c(\mathrm{NaCl})=250 \mathrm{mM}$

$\mathrm{IC}_{50}=4.3 \mu \mathrm{M}$

Stöchiometrie: 6:1 Polymer 1:Lysozyme

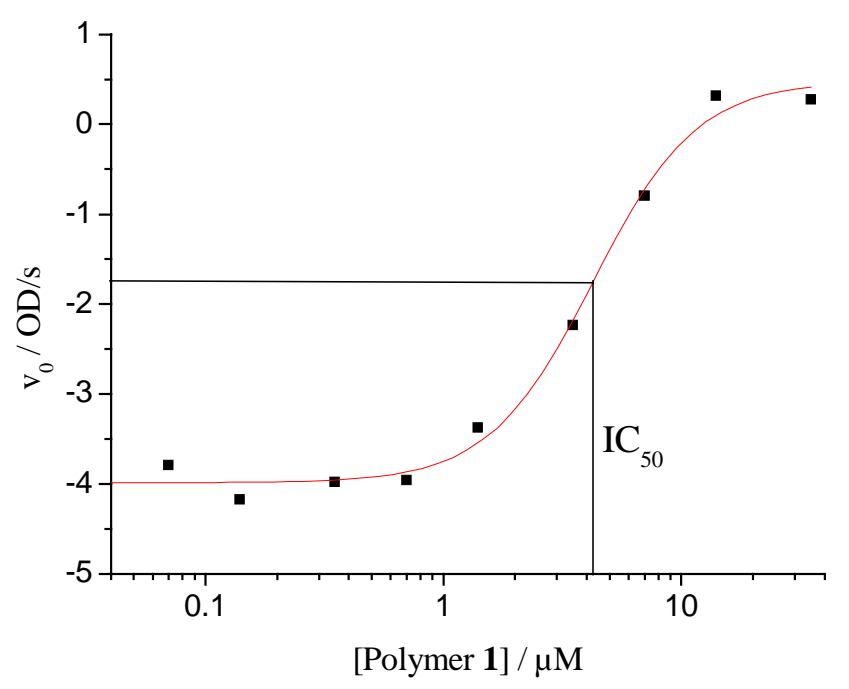

\begin{tabular}{|c|c|}
\hline [Polymer 1] $/ \mu \mathrm{M}$ & $\mathrm{v}_{0} / \mathrm{OD} / \mathrm{s}$ \\
\hline 34.988 & 0.27 \\
\hline 13.995 & 0.32 \\
\hline 6.998 & -0.80 \\
\hline 3.499 & -2.24 \\
\hline 1.400 & -3.38 \\
\hline 0.700 & -3.96 \\
\hline 0.350 & -3.98 \\
\hline 0.140 & -4.17 \\
\hline 0.070 & -3.79 \\
\hline 0.035 & -3.69 \\
\hline 0.014 & -4.01 \\
\hline 0.007 & -4.26 \\
\hline
\end{tabular}




\section{SDS PAGE.}

Laemmli procedure: Gels containing $7.5 \%$ (stacking gel) and $17.5 \%$ (separation gel) acrylamide were prapared from a stock solution of $30 \%$ by weight of acrylamide and $1 \%$ by weight of $N, N$ '-bis-methylene acrylamide. The final concentrations in the separation gels were as follows: $0.375 \mathrm{M}$ Tris- $\mathrm{HCl}(\mathrm{pH} \mathrm{8.8)}$ and $0.1 \%$ SDS. Ther gels were polymerized chemically by adding $0.5 \%$ tetramethylethylenediamine (TEMED) and ammonium persulphate. The $7.5 \%$ stacking gels contained $0.375 \mathrm{M}$ Tris- $\mathrm{HCl}(\mathrm{pH} 6.8)$ and $0.1 \%$ SDS were chemically polymerized the same way as for the saparating gel.

The electrode buffer contained $25 \mathrm{mM}$ Tris, $192 \mathrm{mM}$ glycine and 0.1\% SDS. $62.5 \mathrm{mM}$ Tris$\mathrm{HCl}$ (pH 6.8), $2 \%$ SDS, $10 \%$ glycerol, $5 \%$ 2-mercaptoethanol and $0.001 \%$ bromophenol blue were used as the sample buffer.

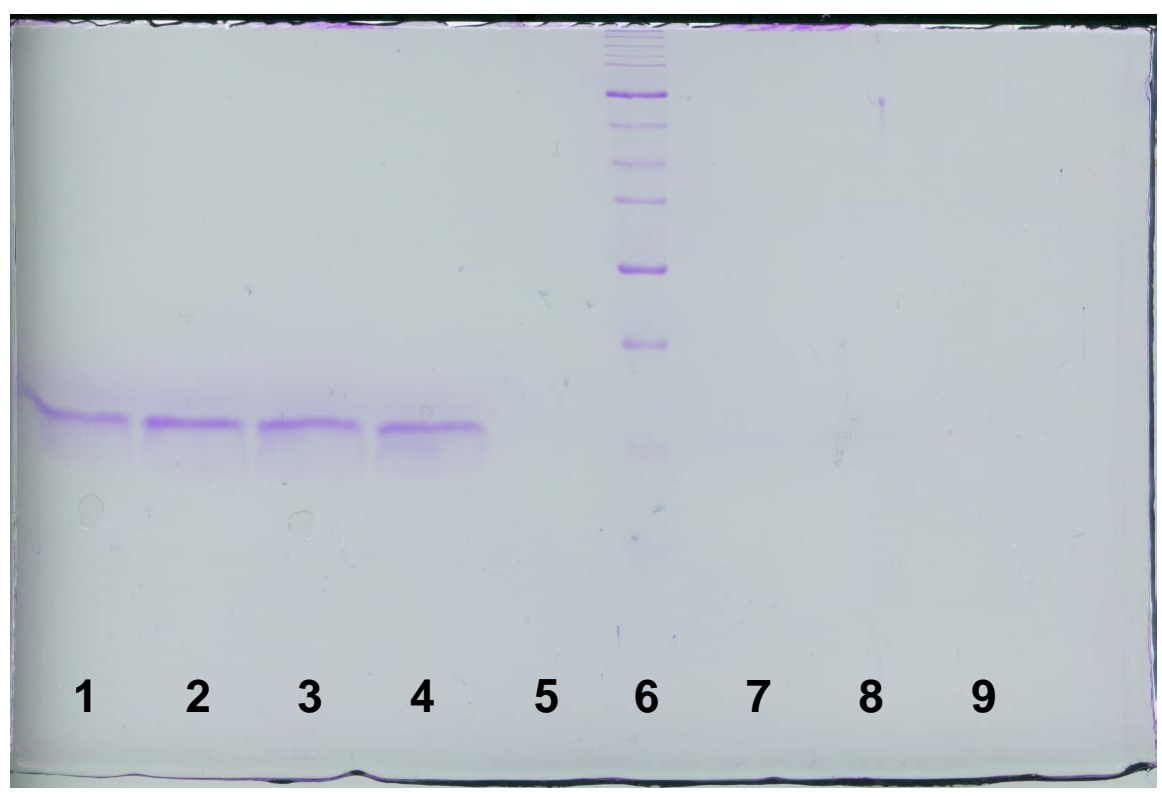

1: pure Lysozyme, 2: Lysozyme +0.5 eq polymer 1, 3: Lysozyme +1 eq polymer 1, 4: Lysozyme +2 eq polymer 1, 5: pure polymer 1, 6: marker, 7-9: potential redissolved pellets of samples 2-4

Contrary to the nondenaturing gel electrophoresis, all samples with Lysozyme produced the same bands in the SDS PAGE, due to the action of the added detergent SDS. However, traces 2-4 together with the empty traces 7-9 prove, that even in the polymer complexes the protein was fully retained in solution. The polymer itself was invisible to Coomassie Blue staining (trace5). 
CD spectra. Unfortunately, CD spectra could not be obtained in the same range as before in the titrations, since the large excess of denaturation agent (usually $1 \mathrm{M}$ ) caused the internal voltage of the $\mathrm{CD}$ instrument to go beyond the acceptable limits. We nevertheless present these spectra below. However, even in the range down to $210 \mathrm{~nm}$ it becomes clear, that lysozyme is not denatured thermally up to $60^{\circ} \mathrm{C}$ (a), but is efficiently denatured by $1 \mathrm{M}$ urea or $1 \mathrm{M} \mathrm{HCl}$ or $5 \mathrm{~h}$ ultrasonic (b, see below). In this case we present 3 flow charts, depicting the $\mathrm{CD}$ spectrum (top), the internal voltage (center), and the UV-vis absorption spectrum (bottom).

a) Attempted thermal denaturation $\left(20^{\circ} \mathrm{C}-60^{\circ} \mathrm{C}\right)$ :

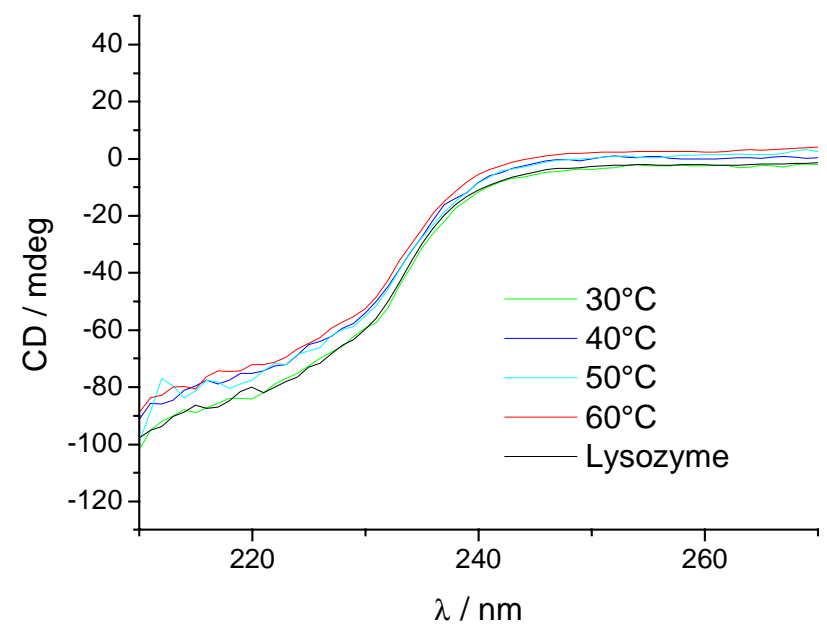


b) Chemical denaturation ( $1 \mathrm{M}$ urea, $1 \mathrm{M} \mathrm{HCl}$, 5h ultrasonic):
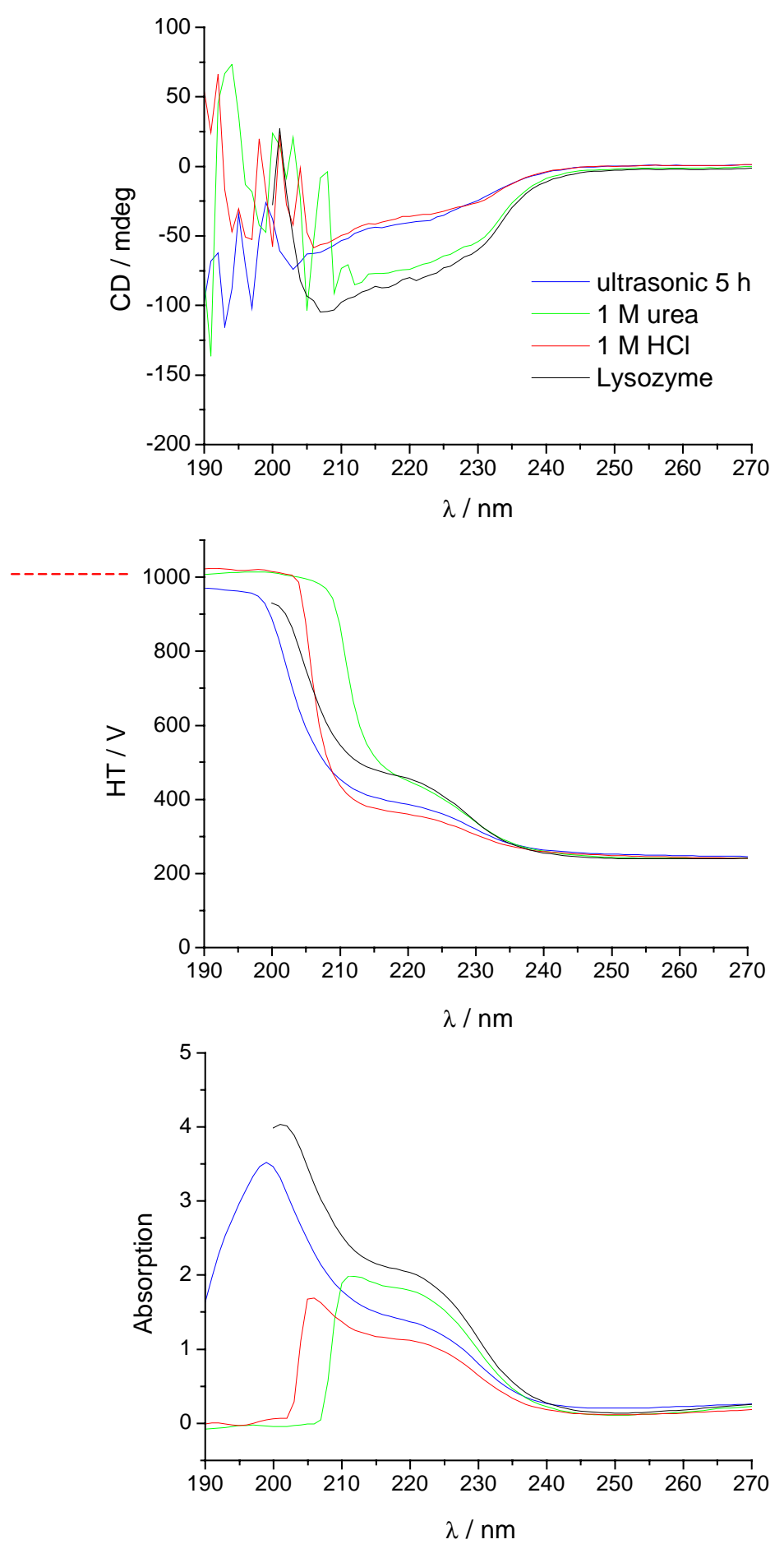


\section{Gel Electrophoresis.}

Gel Electrophoresis was initally carried out under conditions similar to the enzyme assay, in a $50 \mathrm{mM}$ phosphate buffer. The same gel electrophoresis was later carried out at higher ionic strength $(150 \mathrm{mM})$; however, under these conditions, the complex of polymer with lysozyme did not migrate at all, even after $2 \mathrm{~h}$. After several repeated experiments with essentially the same outcome, it was tentatively concluded that contrary to free aqueous solution, the environment of the agarose gel leads to immobilization of the complex, most likely due to a noncovalent interaction with agarose.

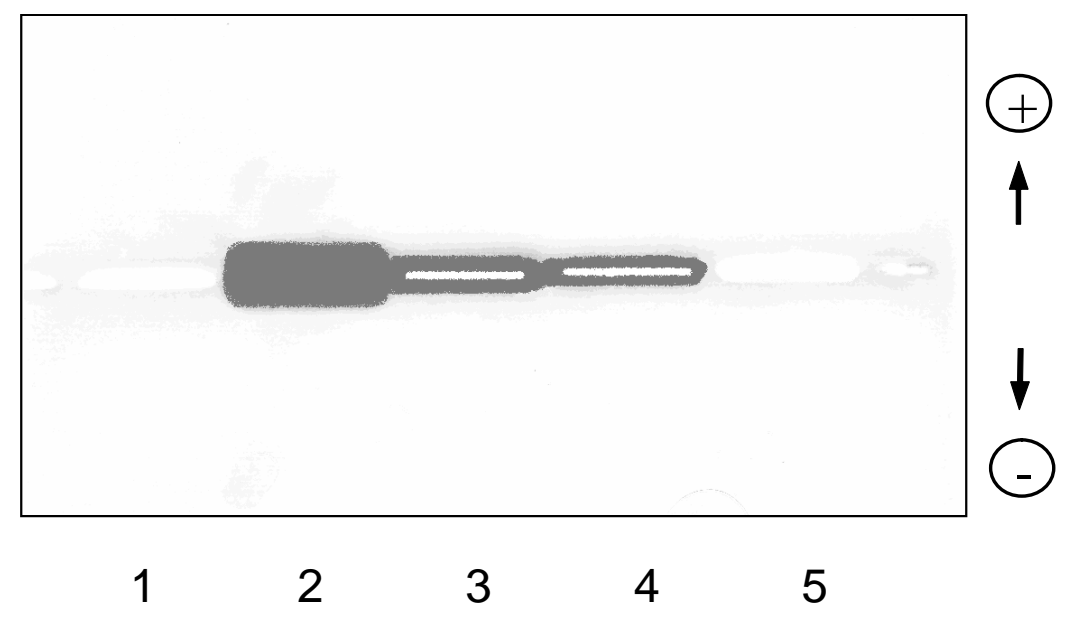

1: Lysozyme; 2: Lysozyme + 1 eq polymer 1; 3: Lysozyme + 2 eqs polymer 1: 4: Lysozyme +10 eqs polymer 1 ; 5 : polymer $\mathbf{1}$. 